\title{
BRANCHING AND GENERALIZED-RECURSIVE INSET ENTROPIES
}

\author{
BRUCE R. EBANKS
}

\begin{abstract}
In the framework of the mixed theory of information, the general form of branching entropies is determined. This result then leads to a characterization of regular, generalized-recursive entropies of randomized systems of events.
\end{abstract}

1. Introduction. A series of papers ([3], [1], [4], [8], [9]) by Aczél, Daróczy, and Kannappan has initiated the study of a new, mixed (as distinguished from probabilistic and nonprobabilistic) theory of information. Therein, entropies (and other measures of information) depend not only upon the probabilities of the events, but also directly upon the events themselves.

Let $X$ be a ring of sets (containing, with any two sets, their union and difference, hence also their intersection and the empty set 0 ). A sequence of maps $I_{n}$ : $X^{n} \times p(X)^{n} \rightarrow R(n=2,3, \ldots ; R$ the set of reals; $p: X \rightarrow[0,1]$ a probability measure) will be called an entropy of randomized systems of events, or simply inset entropy. We seek inset entropies which satisfy certain desirable properties, analogous to those in the probabilistic theory of information (cf. Aczél-Daróczy [2]).

In the probabilistic theory, $\mathrm{C}$. $\mathrm{T}$. $\mathrm{Ng}$ [7] has characterized branching, symmetric entropies as exactly those which are representable as a sum. A similar result has been proven by the author [5] for entropies of sequences of elements of a semigroup. In the present setting of the mixed theory of information, an inset entropy $I_{n}(n=2,3, \ldots)$ is said to be branching if there exist maps $\Delta_{n, i}: X^{3} \times$ $p(X)^{3} \rightarrow R(i=1,2, \ldots, n-1)$ such that, for all $\left(x_{1}, x_{2}, \ldots, x_{n}\right) \in X^{n}$,

$$
\begin{aligned}
& I_{n}\left(\begin{array}{ccc}
x_{1}, & x_{2}, \ldots, & x_{n} \\
p\left(x_{1}\right), & p\left(x_{2}\right), \ldots, & p\left(x_{n}\right)
\end{array}\right) \\
& \quad=I_{n}\left(\begin{array}{cccc}
x_{1}, \ldots, x_{i-1}, & x_{i} \cup x_{i+1}, & 0, & x_{i+2}, \ldots, x_{n} \\
p\left(x_{1}\right), \ldots, p\left(x_{i-1}\right), & p\left(x_{i} \cup x_{i+1}\right), & 0, & p\left(x_{i+2}\right), \ldots, p\left(x_{n}\right)
\end{array}\right) \\
& \quad+\Delta_{n, i}\left(\begin{array}{ccc}
x_{i}, & x_{i+1}, & x_{i} \cup x_{i+1} \\
p\left(x_{i}\right), & p\left(x_{i+1}\right), & p\left(x_{i} \cup x_{i+1}\right)
\end{array}\right) .
\end{aligned}
$$

Received by the editors June 8, 1979; presented to the Society, January 7, 1978 under the title Information measures depending directly on events.

AMS (MOS) subject classifications (1970). Primary 94A15, 39A30, 39A20.

Key words and phrases. Inset entropy, branching, randomized system of events, mixed theory of information, generalized recursivity. 
THEOREM 1.1. $I_{n}: X^{n} \times p(X)^{n} \rightarrow R(n=2,3, \ldots)$ is (1.1) branching if, and only if, there exist maps $\phi_{n, i}: X \times p(X) \rightarrow R(i=0,1, \ldots, n)$, such that, for all $\left(x_{1}, x_{2}, \ldots, x_{n}\right) \in X^{n}$,

$$
\begin{gathered}
I_{n}\left(\begin{array}{ccc}
x_{1}, & x_{2}, \ldots, & x_{n} \\
p\left(x_{1}\right), & p\left(x_{2}\right), \ldots, & p\left(x_{n}\right)
\end{array}\right) \\
=\sum_{i=1}^{n} \phi_{n, i}\left(x_{i}, p\left(x_{i}\right)\right)+\phi_{n, 0}\left(\bigcup_{i=1}^{n} x_{i}, p\left(\bigcup_{i=1}^{n} x_{i}\right)\right), \\
\phi_{n, i}(0,0)=0 \text { for } i=1,2, \ldots, n .
\end{gathered}
$$

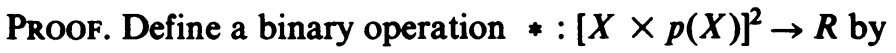

$$
(x, p(x)) *(y, p(y)):=(x \cup y, p(x \cup y))
$$

for all $x, y \in X$. Since $(X \times p(X)$, $)$ is an idempotent, abelian monoid (with unity $(0,0)$ ), Theorem 3.2 of [5] gives the complete solution of (1.1) as (1.2).

We now examine a special form of (1.1) which $I_{n}$ may satisfy if the $x_{i}$ 's are disjoint. Let

$$
X_{n}:=\left\{\left(x_{1}, x_{2}, \ldots, x_{n}\right) \in X^{n} \mid x_{i} \cap x_{j}=0 \text { for } i \neq j ; i, j=1,2, \ldots, n\right\}
$$

and

$$
\Gamma_{n}:=\left\{\left(p_{1}, p_{2}, \ldots, p_{n}\right) \mid \sum_{i=1}^{n} p_{i}=1 ; p_{i}>0 \text { for } i=1,2, \ldots, n\right\}
$$

for all $n=2,3, \ldots$ An inset entropy $I_{n}(n=2,3, \ldots)$ is said to be generalizedrecursive if

$$
\begin{aligned}
I_{n}\left(\begin{array}{c}
x_{1}, x_{2}, \ldots, x_{n} \\
p_{1}, p_{2}, \ldots, p_{n}
\end{array}\right)= & I_{n-1}\left(\begin{array}{cc}
x_{1} \cup x_{2}, x_{3}, \ldots, x_{n} \\
p_{1}+p_{2}, p_{3}, \ldots, p_{n}
\end{array}\right) \\
& +g\left(p_{1}+p_{2}\right) I_{2}\left(\begin{array}{cc}
x_{1}, & x_{2} \\
p_{1} / p_{1}+p_{2}, & p_{2} / p_{1}+p_{2}
\end{array}\right)
\end{aligned}
$$

whenever $p_{1}+p_{2}>0$, for all

$$
\left(\begin{array}{l}
x_{1}, x_{2}, \ldots, x_{n} \\
p_{1}, p_{2}, \ldots, p_{n}
\end{array}\right) \in X_{n} \times \Gamma_{n} \quad(n=2,3, \ldots),
$$

where $p_{i}=p\left(x_{i}\right)$ and $g:[0,1] \rightarrow R$ satisfies

$$
g(p q)=g(p) g(q), \quad \forall p, q \in] 0,1]
$$

This property generalizes both the recursivity (in which $g(p) \equiv p$ ) introduced in [3] and the $\alpha$-recursivity (in which $g(p) \equiv p^{\alpha}$ ) introduced in [4].

Generalizing a probabilistic result of the author [6], all regular, symmetric, expansible, branching entropies which are also generalized-recursive are determined. In the probabilistic setting, the entropies determined are essentially the Shannon entropy, defined on $\Gamma_{n}$ by

$$
H_{n}\left(p_{1}, p_{2}, \ldots, p_{n}\right):=-\sum_{i=1}^{n} p_{i} \log _{2} p_{i} \quad\left(0 \log _{2} 0:=0\right),
$$


and entropies of degree $\alpha \neq 1$,

$$
H_{n}^{\alpha}\left(p_{1}, p_{2}, \ldots, p_{n}\right):=\left(2^{1-\alpha}-1\right)^{-1}\left(\sum_{i=1}^{n} p_{i}^{\alpha}-1\right) \quad\left(0^{\alpha}:=0\right),
$$

$\left(p_{1}, p_{2}, \ldots, p_{n}\right) \in \Gamma_{n} ; n=2,3, \ldots$ We shall see these again as parts of generalized-recursive inset entropies.

Remark 1.2. In [3], [1], [4], inset entropies are defined only on $X_{n} \times \Gamma_{n}$. If entropies are to be defined only on this restricted domain, then representation (1.2) can be assumed initially (on $X_{n} \times \Gamma_{n}$ ) instead of the (1.1) branching property. Of course it would be desirable to have a Theorem 1.1 on $X_{n} \times \Gamma_{n}$, but this seems to be very difficult. Note also that the $p_{i}$ are independent of the $x_{i}$; the notations $p\left(x_{i}\right)$, $p(x)$ are used initially partly because $\sum_{i=1}^{n} p\left(x_{i}\right) \geqslant 1$ is possible.

2. Main result. First, we have an easy consequence of Theorem 1.1. An entropy $I_{n}(n=2,3, \ldots)$ is symmetric if, for all permutations $\pi$ on $(1,2, \ldots, n)$ and all $\left(\begin{array}{l}x_{i} \\ p_{i}\end{array}\right)_{i} \in X^{n} \times[0,1]^{n}$,

$$
I_{n}\left(\begin{array}{l}
x_{1}, x_{2}, \ldots, x_{n} \\
p_{1}, p_{2}, \ldots, p_{n}
\end{array}\right)=I_{n}\left(\begin{array}{l}
x_{\pi(1)}, x_{\pi(2)}, \ldots, x_{\pi(n)} \\
p_{\pi(1)}, p_{\pi(2)}, \ldots, p_{\pi(n)}
\end{array}\right),
$$

and expansible if, for all $\left(\begin{array}{c}x_{i} \\ p_{i}\end{array}\right)_{i} \in X^{n} \times[0,1]^{n}$,

$$
I_{n+1}\left(\begin{array}{l}
x_{1}, x_{2}, \ldots, x_{n}, 0 \\
p_{1}, p_{2}, \ldots, p_{n}, 0
\end{array}\right)=I_{n}\left(\begin{array}{c}
x_{1}, x_{2}, \ldots, x_{n} \\
p_{1}, p_{2}, \ldots, p_{n}
\end{array}\right) .
$$

Corollary 2.1. $I_{n}: X^{n} \times[0,1]^{n} \rightarrow R(n=2,3, \ldots)$ is (1.1) branching, (2.1) symmetric, and (2.2) expansible if, and only if, there exist maps $\phi, \phi_{0}: X \times[0,1] \rightarrow R$ such that, for all $\left(\boldsymbol{x}_{p_{i}}\right)_{i} \in X^{n} \times[0,1]^{n}$,

$$
\begin{gathered}
I_{n}\left(\begin{array}{l}
x_{1}, x_{2}, \ldots, x_{n} \\
p_{1}, p_{2}, \ldots, p_{n}
\end{array}\right)=\sum_{i=1}^{n} \phi\left(x_{i}, p_{i}\right)+\phi_{0}\left(\bigcup_{i=1}^{n} x_{i}, p\left(\bigcup_{i=1}^{n} x_{i}\right)\right) \\
\phi(0,0)=0 .
\end{gathered}
$$

Proof. Theorem 1.1 and [5, Corollary 7.5].

In view of Corollary 2.1, we state the principal result as follows.

THEOREM 2.2. An inset entropy $I_{n}(n=2,3, \ldots)$ is $((1.3),(1.4))$ generalized-recursive and has the (2.3) sum representation on $X_{n} \times \Gamma_{n}$ with (2.3A) and

$$
\left.p \mapsto \phi(x, p) \in \mathcal{L}^{1}\right] 0,1[\quad(\forall x \in X)
$$

if, and only if, it is representable, for all $\left(\left(_{p_{i}}^{x_{i}}\right)_{i} \in X_{n} \times \Gamma_{n}\right.$, in one of the forms

$$
\begin{gathered}
I_{n}\left(\begin{array}{c}
x_{1}, x_{2}, \ldots, x_{n} \\
p_{1}, p_{2}, \ldots, p_{n}
\end{array}\right)=\gamma H_{n}\left(p_{1}, p_{2}, \ldots, p_{n}\right)+\sum_{i=1}^{n} p_{i} b\left(x_{i}\right)-b\left(\bigcup_{i=1}^{n} x_{i}\right) \\
I_{n}\left(\begin{array}{c}
x_{1}, x_{2}, \ldots, x_{n} \\
p_{1}, p_{2}, \ldots, p_{n}
\end{array}\right)=\sum_{i=1}^{n} p_{i}^{\alpha} a\left(x_{i}\right)-a\left(\bigcup_{i=1}^{n} x_{i}\right) \quad(\alpha \neq 0,1) \\
I_{n}\left(\begin{array}{l}
x_{1}, x_{2}, \ldots, x_{n} \\
p_{1}, p_{2}, \ldots, p_{n}
\end{array}\right)=\gamma+f\left(\bigcup_{i=1}^{n} x_{i}\right)
\end{gathered}
$$




$$
I_{n}\left(\begin{array}{l}
x_{1}, x_{2}, \ldots, x_{n} \\
p_{1}, p_{2}, \ldots, p_{n}
\end{array}\right)=\sum_{p_{i} \neq 0} f\left(x_{i}\right)+\sum_{p_{i}=0} h\left(x_{i}\right)-f\left(\bigcup_{i=1}^{n} x_{i}\right),
$$

where $\gamma$ is a constant, $a, b, f, h: X \rightarrow R$ with $h(0)=0$ and $H_{n}$ is defined by (1.5).

Note that (2.4), (2.5) are the same forms found in [3], [4], respectively.

The "if" part of Theorem 2.2 is a simple verification. For the "only if" part, we start with

LEMMA 2.3. If $I_{n}$ is generalized-recursive and has the (2.3) sum representation with $\left.p \mapsto \phi(x, p) \in \mathcal{L}^{1}\right] 0,1[$, then $\phi$ has one of the following four forms (with corresponding $g$ of (1.3)).

$$
\begin{gathered}
\phi(x, p)=a(x) p \log _{2} p+b(x) p-c(x), \quad g(p)=p, \\
\phi(x, p)=a(x) p^{\alpha}+b(x) p-c(x), \quad g(p)=p^{\alpha} \quad(\alpha \neq 1), \\
\phi(x, p)=a(x) p-c(x), \quad g=\text { arbitrary solution of }(1.4)
\end{gathered}
$$

for all $p \in] 0,1]$ and all $x \in X$. Furthermore, provided $g \neq 0$, the $\phi_{0}$ of (2.3) satisfies

$$
\phi_{0}(x, 1)=-\phi(x, 1), \quad \forall x \in X \text {. }
$$

Proof. Substituting (2.3) for $\left(\begin{array}{l}x_{i} \\ p_{i}\end{array}\right)_{i} \in X_{n} \times \Gamma_{n}$ into (1.3), we have (with $p=p_{1}$, $\left.q=p_{2}\right)$,

$$
\begin{aligned}
& \phi\left(x_{1}, p\right)+\phi\left(x_{2}, q\right)-\phi\left(x_{1} \cup x_{2}, p+q\right) \\
& \quad=g(p+q)\left[\phi\left(x_{1}, p / p+q\right)+\phi\left(x_{2}, q / p+q\right)+\phi_{0}\left(x_{1} \cup x_{2}, 1\right)\right],
\end{aligned}
$$

for all $(p, q) \in D:=\{(p, q) \mid p, q \geqslant 0,0<p+q \leqslant 1\}$. Fixing $\left(x_{1}, x_{2}\right) \in X_{2}$ temporarily and defining $h_{1}, h_{2}, h_{3}: X \rightarrow R$ and $\beta \in R$ by

$$
\begin{aligned}
h_{i}(p):=\phi\left(x_{i}, p\right), \quad i=1,2, \quad h_{3}(p):=\phi\left(x_{1} \cup x_{2}, p\right), \\
\beta:=\phi_{0}\left(x_{1} \cup x_{2}, 1\right),
\end{aligned}
$$

we obtain from (2.12), for all $(p, q) \in D$,

$$
h_{1}(p)+h_{2}(q)-h_{3}(p+q)=g(p+q)\left[h_{1}(p / p+q)+h_{2}(q / p+q)+\beta\right] \text {. }
$$

With $p=0$, resp. $q=0,(2.14)$ yields

$$
h_{2}(q)=h_{3}(q)+\delta_{1} g(q)+\delta_{2}, \quad h_{1}(p)=h_{3}(p)+\delta_{3} g(p)+\delta_{4},
$$

for some constants $\delta_{i}(i=1,2,3,4)$. Now (2.14), (2.15), and the (1.4) multiplicativity of $g$ give, for all $(p, q) \in D^{\prime}:=\{(p, q) \mid(p, q) \in D, p q \neq 0\}$,

$$
\begin{aligned}
h_{3}(p)+ & h_{3}(q)-h_{3}(p+q)+\delta_{2}+\delta_{4} \\
& =g(p+q)\left[h_{3}(p / p+q)+h_{3}(q / p+q)+\delta_{2}+\delta_{4}+\beta\right] .
\end{aligned}
$$

Defining $F:[0,1] \rightarrow R$ by

$$
F(p):= \begin{cases}0, & \text { if } p=0, \\ h_{3}(p)+\left(\beta-\delta_{2}-\delta_{4}\right) p+\delta_{2}+\delta_{4}, & \text { if } p \in] 0,1]\end{cases}
$$

we can write (2.16) as

$$
F(p)+F(q)-F(p+q)=g(p+q)[F(p / p+q)+F(q / p+q)]
$$


So far, (2.18) holds only on $D^{\prime}$, but we need it for all $(p, q) \in D$.

To this end, put $q=1-p$ in (2.12). If $g(1)=1$, then (2.11) holds. On the other hand, if $g(1) \neq 1$, then $g \equiv 0$ by (1.4) with $q=1$. Now let us check that (2.18) is true for, say, $p=0$. It is true, by (2.17) and (2.13), if $0=g(q) F(1)=g(q)\left[h_{3}(1)+\right.$ $\beta]=g(q)\left[\phi\left(x_{1} \cup x_{2}, 1\right)+\phi_{0}\left(x_{1} \cup x_{2}, 1\right)\right]$. But this is true since either $g \equiv 0$ or (2.11) holds. Thus we have (2.18) for all $(p, q) \in D$.

Furthermore, by the integrability assumption on $\phi$, and by (2.13) and (2.17), $\left.F \in \mathcal{L}^{1}\right] 0,1[$. Under this condition, the only solutions of (2.18) (with (1.4)) are given by the author [6] as

$$
\begin{gathered}
F(p)=a p \log _{2} p, \quad g(p)=p \quad\left(0 \log _{2} 0:=0\right) ; \\
F(p)=a\left(p^{\alpha}-p\right), \quad g(p)=p^{\alpha} \quad\left(0^{\alpha}:=0, \alpha \neq 1\right) ; \\
F(p)=a p, \quad g(p)=0 ; \\
F(p)=\left\{\begin{array}{lr}
k, & p=0, \\
a(1-p), & p>0,
\end{array} \quad g(p)=1 ;\right. \\
F(p)=0, \quad g=\text { arbitrary solution of (1.4); }
\end{gathered}
$$

for some constants $a, k$, and $\alpha(\neq 1)$. Comparison of these results with (2.17) yields, for suitable constants $a, b, c$, and only for $p \in[0,1]$,

$$
\begin{gathered}
h_{3}(p)=a p \log _{2} p+b p-c, \quad g(p)=p ; \\
h_{3}(p)=a p^{\alpha}+b p-c, \quad g(p)=p^{\alpha} \quad(\alpha \neq 1) ; \\
h_{3}(p)=a p-c, \quad g=\text { arbitrary solution of (1.4). }
\end{gathered}
$$

In view of (2.13), letting $\left(x_{1}, x_{2}\right) \in X_{2}$ vary, the formulas for $h_{3}$ and $g$ give rise to solutions (2.8) through (2.10) for $\phi$ and $g$.

3. Proof of the main result. Theorem 2.2 is established by Lemma 2.3 in conjunction with the three lemmas of this section.

LEMMA 3.1. If $I_{n}(n=2,3, \ldots)$ is generalized-recursive and has the (2.3) sum form on $X_{n} \times \Gamma_{n}$, with $\phi, \phi_{0}, g$ given by (2.8), (2.11), then $I_{n}$ has the form (2.4) for some constant $\gamma$ and map $b: X \rightarrow R$.

Proof. Substituting (2.8) into (2.12), and using (2.11), we get, after some rearrangement,

$$
\begin{aligned}
{\left[c\left(x_{1}\right)+\right.} & \left.c\left(x_{2}\right)-c\left(x_{1} \cup x_{2}\right)\right](p+q-1) \\
& =\left[a\left(x_{1} \cup x_{2}\right)(p+q)-a\left(x_{1}\right) p-a\left(x_{2}\right) q\right] \log _{2}(p+q),
\end{aligned}
$$

for all $(p, q) \in D^{\prime}$ and all $\left(x_{1}, x_{2}\right) \in X_{2}$. Examining terms which are constant (with respect to $p$ and $q$ ) in (3.1), we see that

$$
c\left(x_{1} \cup x_{2}\right)=c\left(x_{1}\right)+c\left(x_{2}\right), \quad \forall\left(x_{1}, x_{2}\right) \in X_{2} .
$$

Now (3.1) gives

$$
a\left(x_{1} \cup x_{2}\right)(p+q)=a\left(x_{1}\right) p+a\left(x_{2}\right) q,
$$

which means that, for $\gamma:=-a(0)$,

$$
a(x)=-\gamma, \quad \forall x \in X .
$$


Now (2.12) with $p=0 \neq q$ yields (by (2.8), (3.2), and (3.3)) $\phi\left(x_{1}, 0\right)+c\left(x_{1}\right)=$ $q\left[\phi\left(x_{1}, 0\right)+c\left(x_{1}\right)\right]$, so that $\phi(x, 0)=-c(x), \forall x \in X$, which is (2.8) for $p=0$. Finally, (2.4) follows from (2.3), (2.8) for all $p \in[0,1],(3.2),(3.3),(2.11)$, and (1.5).

LEMMA 3.2. If $I_{n}(n=2,3, \ldots)$ is generalized-recursive and has the (2.3) sum form on $X_{n} \times \Gamma_{n}$, with $\phi, \phi_{0}, g$ given by (2.9) and (2.11), then $I_{n}$ has either form (2.5) for some map $a: X \rightarrow R$ and constant $\alpha(\neq 0,1)$, or form (2.7) for some maps $f, h$ : $X \rightarrow R$ with $h(0)=0$.

Proof. Substituting (2.9) and (2.11) into (2.12), we get

$$
\begin{aligned}
& {\left[c\left(x_{1} \cup x_{2}\right)-c\left(x_{1}\right)-c\left(x_{2}\right)\right]\left[1-(p+q)^{\alpha}\right]} \\
& \quad=\left[b\left(x_{1} \cup x_{2}\right)(p+q)-b\left(x_{1}\right) p-b\left(x_{2}\right) q\right]\left[1-(p+q)^{\alpha-1}\right]
\end{aligned}
$$

for all $(p, q) \in D^{\prime}$ and all $\left(x_{1}, x_{2}\right) \in X_{2}$. We consider two cases.

Case 1. Suppose $\alpha \neq 0$. We again get (3.2) upon equating constants (in $p$ and $q$ ). Then (3.4) yields (since $\alpha \neq 1$ )

$$
b\left(x_{1} \cup x_{2}\right)(p+q)=b\left(x_{1}\right) p+b\left(x_{2}\right) q .
$$

Equating coefficients of $p$, we obtain $b\left(x_{1} \cup x_{2}\right)=b\left(x_{1}\right)$, so there is a constant $\gamma$ such that

$$
b(x)=\gamma, \quad \forall x \in X .
$$

By (2.9), (3.2), and (3.5), (2.12) with $p=0 \neq q$ becomes $\phi\left(x_{1}, 0\right)+c\left(x_{1}\right)=$ $q^{\alpha}\left[\phi\left(x_{1}, 0\right)+c\left(x_{1}\right)\right]$. Thus we have (2.9) for $p=0$, i.e., $\phi(x, 0)=-c(x), \forall x \in X$. Now (2.5) follows from (2.3), (2.11), (2.9) for all $p \in[0,1],(3.2)$, and (3.5).

Case 2. Suppose $\alpha=0$. Then (3.4) becomes, after multiplication by $(p+q)$,

$$
0=\left[b\left(x_{1} \cup x_{2}\right)(p+q)-b\left(x_{1}\right) p-b\left(x_{2}\right) q\right](p+q-1) .
$$

Examining coefficients of $p^{2}$, we see that $b\left(x_{1} \cup x_{2}\right)=b\left(x_{1}\right)$, so that (3.5) again holds for some constant $\gamma$.

However, (2.9) does not hold for $p=0$. Indeed, $\phi(x, 0)$ is arbitrary, say

$$
h(x):=\phi(x, 0), \quad \forall x \in X .
$$

Also, (3.2) does not hold, so we define $f: X \rightarrow R$ by

$$
f(x):=a(x)-c(x), \quad \forall x \in X .
$$

Finally, (2.7) follows from (2.3), (2.11), (2.9) for $\alpha=0$ and $p \in$ ]0, 1], (3.5), (3.6), and (3.7). Moreover, $h(0)=0$ by (3.6) and (2.3A).

LEMMA 3.3. If $I_{n}(n=2,3, \ldots)$ is generalized-recursive and has the (2.3) sum form on $X_{n} \times \Gamma_{n}$, with $\phi, \phi_{0}, g$ given by (2.10) and (provided $g \neq 0$ ) (2.11), then $I_{n}$ has one of the forms (2.4), (2.6), (2.7).

Proof. We consider three cases.

Case 1. Suppose $g \neq 0$ and $g \neq 1$. By (1.4), this means that $g$ is nonconstant. By (2.10) and (2.11), (2.12) becomes

$$
\begin{aligned}
& {\left[c\left(x_{1} \cup x_{2}\right)-c\left(x_{1}\right)-c\left(x_{2}\right)\right][1-g(p+q)]} \\
& \quad=\left[a\left(x_{1} \cup x_{2}\right)(p+q)-a\left(x_{1}\right) p-a\left(x_{2}\right) q\right]\left[1-g(p+q)(p+q)^{-1}\right]
\end{aligned}
$$


for all $(p, q) \in D^{\prime}$ and for all $\left(x_{1}, x_{2}\right) \in X_{2}$. Since $g$ is nonconstant, we again have (3.2) by equating constants. Moreover, if $g(p) \equiv p$, then $I_{n}$ has the form (2.4) by Lemma 3.1. Otherwise, (3.8) and (3.2) give $a\left(x_{1} \cup x_{2}\right)(p+q)=a\left(x_{1}\right) p+a\left(x_{2}\right) q$, which again means that we have

$$
a(x)=\gamma \text { (constant), } \forall x \in X .
$$

Finally, we again get $\phi(x, 0)=-c(x)$ (i.e., (2.10) for $p=0$ ) by (2.12) with $p=0 \neq q,(2.10),(2.11),(3.9),(3.2)$, and the nonconstance of $g$. By the additional use of (2.3), we find that $I_{n} \equiv 0$, a special case of (2.4), (2.6), or (2.7).

Case 2. Suppose $g \equiv 1$. By (2.10) and (2.11), (2.12) becomes, after multiplication by $(p+q)$,

$$
\left[a\left(x_{1}\right) p+a\left(x_{2}\right) q-a\left(x_{1} \cup x_{2}\right)(p+q)\right](p+q-1)=0
$$

for $(p, q) \in D^{\prime},\left(x_{1}, x_{2}\right) \in X_{2}$. Upon examining the coefficient of $p^{2}$, we find that (3.9) holds once again.

But, as in Case 2 of the proof of Lemma 3.2, $\phi(x, 0)$ is arbitrary, and we define $h: X \rightarrow R$ by (3.6). By (2.3), (2.11), (2.10) for $p \in] 0,1]$, (3.9), and (3.6), we obtain (2.7) with map $f:=-c$.

Case 3. Suppose $g \equiv 0$. By (2.10), (2.12) becomes

$$
a\left(x_{1}\right) p+a\left(x_{2}\right) q-a\left(x_{1} \cup x_{2}\right)(p+q)=c\left(x_{1}\right)+c\left(x_{2}\right)-c\left(x_{1} \cup x_{2}\right),
$$

for $(p, q) \in D^{\prime},\left(x_{1}, x_{2}\right) \in X_{2}$. Again, (3.2) and (3.9) follow.

Next, (2.12) with $p \neq 0=q$ gives $\phi\left(x_{2}, 0\right)=-c\left(x_{2}\right)$, so (2.10) holds for all $p \in[0,1]$. Now, by (2.3), (2.10), (3.2), and (3.9), we get form (2.6) for $I_{n}$, where $f(x):=-c(x)+\phi_{0}(x, 1)$.

4. Remarks. There is an analytic connection between (2.4) and (2.5). If $b: X \rightarrow R$ is defined by

$$
b(x):=a(x)-\gamma\left(2^{1-\alpha}-1\right)^{-1}, \quad \forall x \in X,
$$

then (2.5) goes over into

$$
\begin{aligned}
I_{n}\left(\begin{array}{c}
x_{1}, x_{2}, \ldots, x_{n} \\
p_{1}, p_{2}, \ldots, p_{n}
\end{array}\right)= & \gamma H_{n}^{\alpha}\left(p_{1}, p_{2}, \ldots, p_{n}\right) \\
& +\sum_{i=1}^{n} p_{i}^{\alpha} b\left(x_{i}\right)-b\left(\bigcup_{i=1}^{n} x_{i}\right),
\end{aligned}
$$

where $H_{n}^{\alpha}: \Gamma_{n} \rightarrow R$ is the entropy of degree $\alpha$, defined in (1.6). As $\alpha$ tends to 1 , the entropy in (4.1) tends to (2.4).

Finally, the results of Theorem 2.2 can be extended, with modifications, to the domain $X^{n} \times[0,1]^{n}$. The representations on this larger domain should, in general, contain the term $\phi_{0}\left(\cup_{i=1}^{n} x_{i}, p\left(\cup_{i=1}^{n} x_{i}\right)\right)$ and not make use of (2.11).

The author wishes to thank the referee for his helpful suggestions.

\section{REFERENCES}

1. J. Aczél, A mixed theory of information. II: Additive inset entropies (of randomized systems of events) with measurable sum property, Utilitas Math. 13 (1978), 49-54.

2. J. Aczél and Z. Daróczy, On measures of information and their characterizations, Academic Press, New York and London, 1975. 
3. , A mixed theory of information. I: Symmetric, recursive and measurable entropies of randomized systems of events, Rev. Française Automat. Informat. Recherche Opérationnelle Sér. Rouge Informat. Théor. 12 (1978), 149-155.

4. J. Aczél and PI. Kannappan, A mixed theory of information. III: Inset entropies of degree $\beta$, Information and Control 39 (1978), 315-322.

5. B. Ebanks, Branching measures of information on strings, Canad. Math. Bull. (to appear).

6. Generalized-recursive entropies (to appear).

7. C. $\mathrm{T}$. $\mathrm{Ng}$, Representation for measures of information with the branching property, Information and Control 25 (1974), 45-56.

8. J. Aczél and P1. Kannappan, On some symmetric recursive inset measures of information, Transactions of the Prague Conference on Information Theory, Statistical Decision Functions and Random Processes, Academia, Prague, 1978.

9. PI. Kannappan, A mixed theory of information. IV: Inset-inaccuracy and directed divergences, Metrika (to appear).

Department of Mathematics, Texas Tech University, Lubbock, Texas 79409 